\title{
Design Analysis of a Dual Rotor Permanent Magnet Machine driven Electric Vehicle
}

\author{
Mohd Izzat Bin Zainuddin ${ }^{1}$, Aravind $\mathrm{CV}^{1, *}$ \\ ${ }^{1}$ School of Engineering, Taylor's University, Malaysia
}

\begin{abstract}
Electric bike in urban countries such as Europe and China commonly used the brushless direct current machine (BLDC) as it able to produce high torque to transport the user from one place to another. However, BLDC torque density can't be improving due to limitation magnetic flux generated by the permanent magnet. Therefore, the performance of electric bike can't be improved. Outer rotor BLDC machine design able to improve the torque density of the motor due to increase radius of the motor which can be explained by simple physics equation (Torque $=$ Force $\mathrm{x}$ radius). However, an outer rotor machine only generates constant speed, which is not suitable for operating under tractive load condition, especially electric bike. The proposed model is a new novel of double layer outer rotor BLDCPM machine which able to amplify the magnetic flux density and improve the torque density of the machine. The mutual magnetic coupling between the inner and outer rotor of the proposed model increase the magnetic flux intensity as both of them acts as individual parts. Thus, the magnetic flux generated by both rotors are double which resulted in improving the performance of the E-bike. Designing parameters and analysing the performance of the proposed 2D model is done using FEA tools. Evaluation of the conventional and proposed model by comparing torque performance, magnetic flux density and motor constant square density. Other than that, speed torque graph also is evaluated to justify either it can operate similarly to ICE engine with gears. Two model is designed which is Single Outer Rotor Brushless Direct Current (SORBLDC) and Double Outer Rotor Brushless Direct Current (DORBLDC) operated with the same cases of 27 Amp current supplied to it and operate under various speed from $500 \mathrm{rpm}$ to $2000 \mathrm{rpm}$. The average torque produce by the conventional and proposed model are $2.045439 \mathrm{Nm}$ and $3.102648 \mathrm{Nm}$. Furthermore, improvement of the proposed model to conventional model in terms of motor constant square density by $24.92 \%$. Therefore, the proposed model able to improve the magnetic flux by amplifying which resulted to increase the torque density of the machine. Furthermore, the speed-torque graph of the proposed machine shows similarity with speed torque graph of ICE engine. Thus, the proposed machine is suitable to operate for bike application
\end{abstract}

\footnotetext{
${ }^{1}$ Corresponding author: aravindcv@ieee.org
} 


\section{Introduction}

BLDC motor consist rotor embedded with permanent magnet and stator coils to produce high torque density. Furthermore, the rotor of the BLDC motor is supplied with direct current (DC) supply and it generates AC voltage waveform with a trapezoidal shape which reduces the total harmonic distortion (THD) in the machine[1]. The reason BLDC machine used for the electric vehicle due to the power supplied to the machine have lesser noise and provide better performance of the motor. However, room for improvement in terms of torque density of the machine need to be done due to the magnetic flux generated by the machine is fixed. In order to increase the magnetic flux the ratio of the permanent magnet needed to be doubled [2]. To achieve more powerful motor, the design of the motor and position along with the angle of the permanent magnet is research in order to obtain better torque density. Having a good design which included the position and angle of the interior permanent magnet in rotor section contribute to increasing the power density of the motor[3]. However, big spaces needed to insert the slanted PM even though an outer rotor machine. Other than that, the torque density of a motor is improved by increasing the radius of the rotor section. The performance of the motor can also be increased by designing new motor structure with double layer outer rotor PM. Mutual magnetic coupling between inner rotor with outer rotor improves magnetic flux intensity by making both of them as an individual part of the machine[4]. The magnetic loading of the machine increased as the flux generated by the permanent magnet is amplified. Thus, the torque density of the motor is increased. Therefore, the objective is to experiment double outer rotor layer machine that amplifies the magnetic flux which configuration on the magnetic flux density to determine the torque density of the motor.

\subsection{Design Concept}

A load of the machine for this research is a tractive load which to be specified is the electric bike. Tractive study is applied in order to analyze and predict the actual speed of the machine for many types of road conditions. In order to reach full capacity in working principle of an electric or hybrid vehicle, electric motor and generator machine are optimized fully in several road conditions. There are 6 conditions faced by the electric vehicle especially electric bike which are an early start, normal speed, acceleration, high speed, deceleration and stop position. Figure 1 shows the diagram of operating conditions faced by the machine[5].

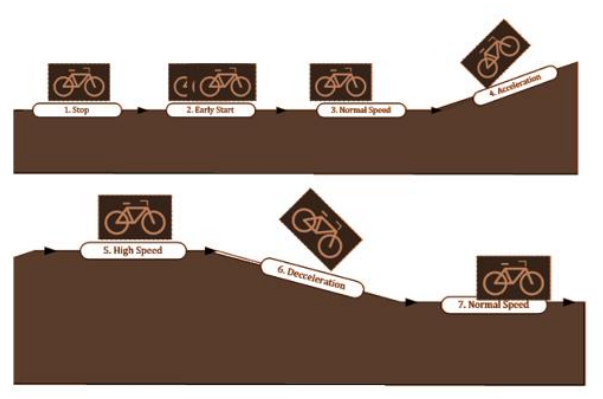

Fig. 1. Operating characteristic of E-bike for all conditions

E-bike is small transportation vehicle where the load on the propulsion unit is traction load. The loading characteristics of the proposed machine must show similarities with ICE 
engine. The speed-torque graph of ICE engine contains 4 parts which each of the section has its own torque and speed characteristics. Figure 2 shows the speed torque graph and speed power graph of ICE engine.

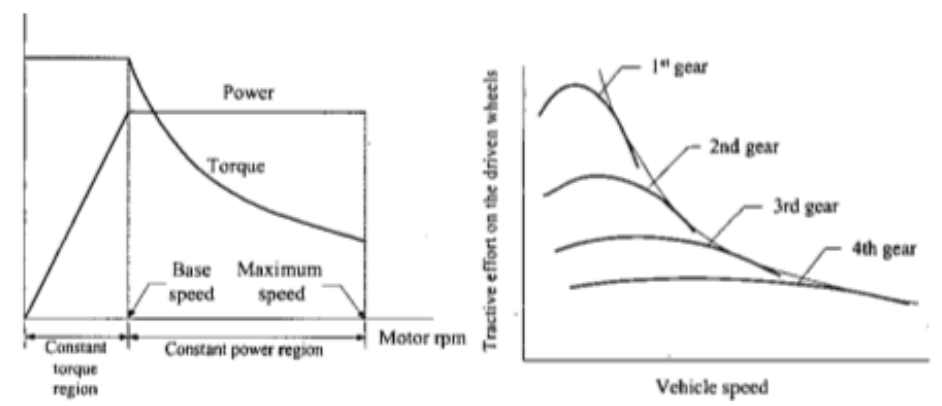

Fig. 2. Speed torque graph and speed power graph of ICE engine

In order to identify the research gap, few motor design had been researching and each of them has its own benefit and drawback. Induction motor widely uses for electric vehicle application high efficiency and low cost. The desired torque of certain speed can be controlled with an inverter can be achieved. However, control schemes of the torque are relatively difficult to control and the constant power range is also limited[6]. Permanent Magnet Synchronous Motor (PMSM) machine has a similar structure to BLDC and induction machine. Advantages PMSM machine are eddy current loss is reduced due to the segmented magnet at the rotor section thus torque density is improved. However, in order for improvement torque density of the machine, the volume of the machine needed to increased especially ratio of the segmented magnet in the rotor section[7]. Another design machine, using the in-wheel motor to SORBLDC machine to improve the magnetic flux by applying the concept of non-contact magnetic planetary gear with the permanent magnet outer rotor BLDC machine. With this method, the transmission torque is maximized resulted to increase the performance of the machine[8]. Coil Testing Study is also done on the SORBLDC in order to study electromagnetic flux generated by the coil windings which effect torque density of the machine. There are two types of armature coil winding implemented on the outer rotor synchronous machine which are concentrated winding and distributed winding. Finite element Analysis using JMAG application is done to simulate both coil winding technique on the conventional model for EV application. The output of the machine for each type of windings are analyzed using FEA tool in order to observer the magnetic field flow and flux density. The winding method that increases the performance of the machine in terms of magnetic field density is chosen. Concentrated windings generate higher peak and saturated flux across the model [9]. Thus, the torque density can be increase due to the magnetic flux generated by the permanent magnet and coil winding in the stator slot. Therefore, magnetic loading is focused for the proposed model on increasing the performance of the machine without changing the output rating of the motor for electric bike application. The proposed design is conducted by applying extra rotor layer to SORBLDC in order to create mutual magnetic coupling between outer rotor with the inner rotor to improve magnetic flux intensity by making both of them as an individual part of the machine. Therefore, the magnetic flux generated by both of individual part is amplified and the performance of the machine improved. Other than that, the proposed model of this research study able to achieve various speed with constant power and torque region[10]. 
The design proposed model is obtained by calculating parameters design formulas in order to know the size of each part. The magnetic circuit is redesigned in order to increase the magnetic flux in order to improve the torque density of the machine. By using FEA tools, design structure of conventional and proposed model is done. Other than that, evaluation of conventional and proposed model is done in terms of magnetic flux density, torque performance, motor constant square density, and power efficiency are done in FEA tools. Speed torque graph and speed power graph of both model is compared to ICE engine application in order to check either they are suitable for bike application. CAD tools are used to design the proposed model so that fabrication of the model can be done.

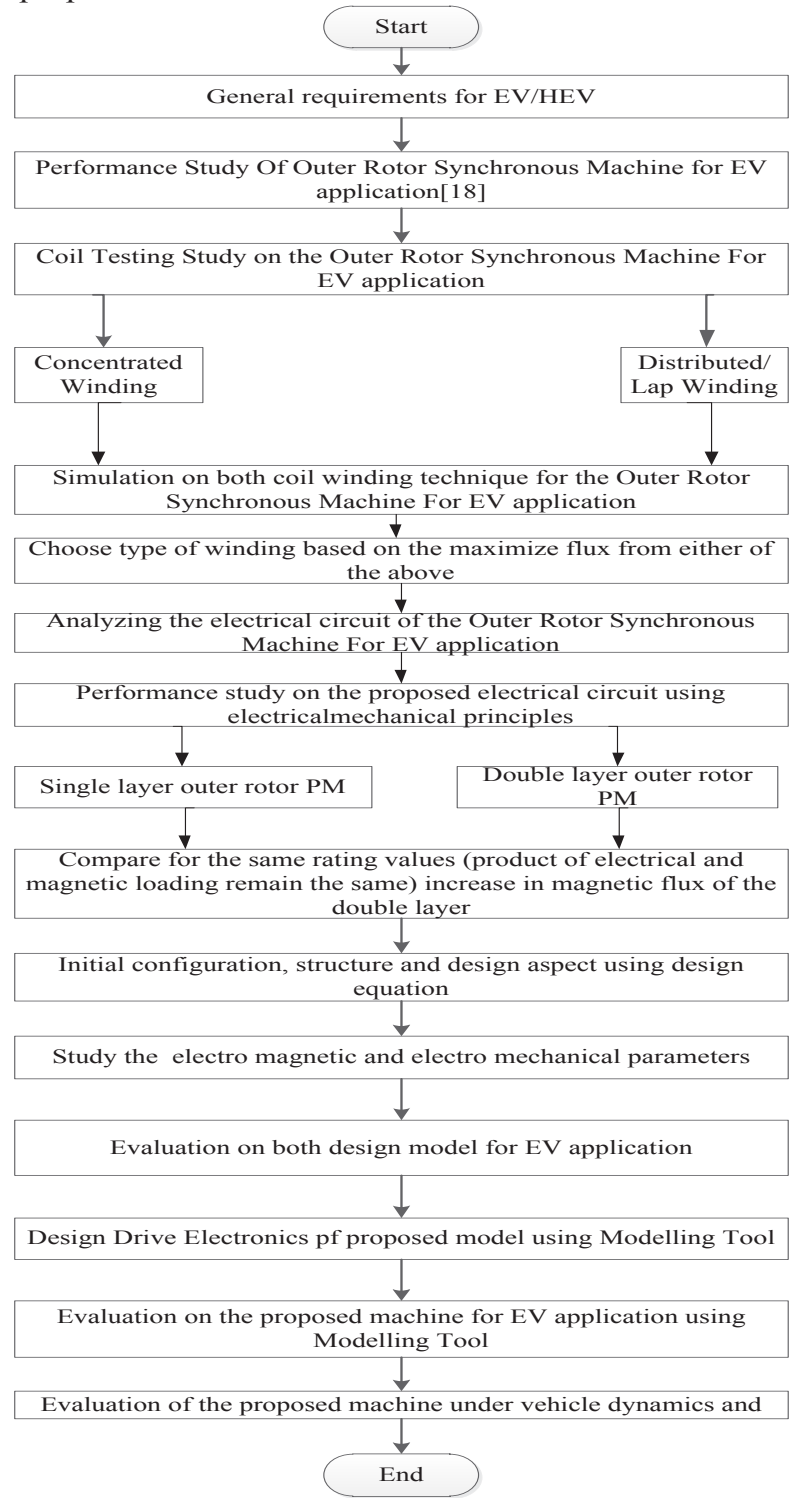

Fig. 4. Methodology used in this research 


\subsection{Proposed Design}

Figure 5(a) shows the 2D proposed DORBLDC model design using JMAG software and Figure 6 shows the exploded view of the proposed DORBLDC model for electric bike application using Solidworks software. The proposed machine contains double layer rotor with a surface permanent magnet that supplied with 3 phase armature coil concentrated windings, 20 permanent magnets mounted on the inner surface rotor and 40 permanent magnets mounted on the outer surface rotor. The proposed model has more benefits than the conventional model as the flux is amplified which resulted to improve flux density across the machine. Table 1 shows the parameter dimensions of the proposed DORBLDC model where the outer dimension of both models are kept the same in order to compare the motor constant square density, magnetic flux density, and torque density

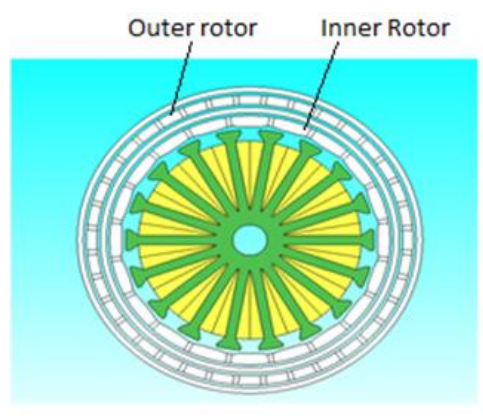

(a) Proposed 2D model

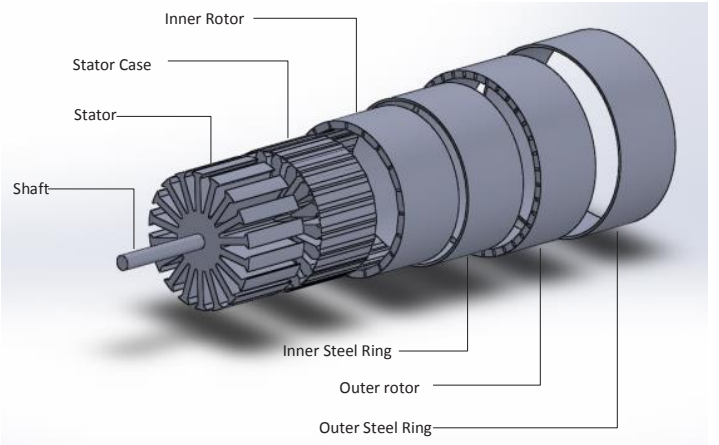

(b) Exploded view

Fig. 5. The Proposed 2D model

Table 1. Specification of the proposed model

\begin{tabular}{|l|l|}
\hline Parameter & Value $(\mathrm{mm})$ \\
\hline Diameter of the machine & 50 \\
\hline Stack length & 50 \\
\hline Number of stator poles & 18 \\
\hline Stator Diameter & 36 \\
\hline Physical Radial Air gap & 1 \\
\hline Outer Ring Diameter & 1.7 \\
\hline Inner ring diameter & 1.7 \\
\hline Inner rotor diameter & 3.523 \\
\hline Outer rotor diameter & 3 \\
\hline Inner PM diameter & 3 \\
\hline Outer PM diameter & 3 \\
\hline Number of poles Inner magnet & 18 \\
\hline Number of poles Outer magnet & 36 \\
\hline
\end{tabular}




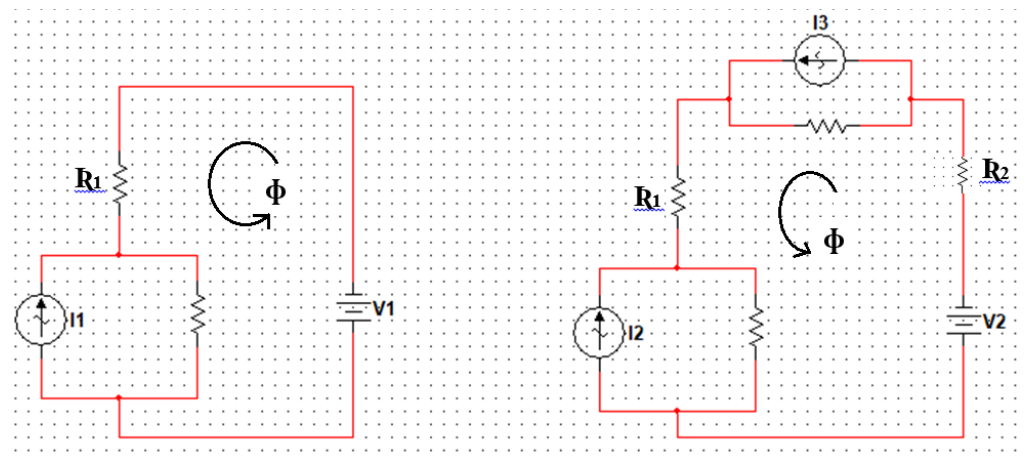

a)Conventional Model

b)Proposed Model

Fig. 6. Magnetic Circuit of Conventional and Proposed Model

$$
\begin{gathered}
\phi=(m m f+F m) / R \\
\phi=(m m f+F m 1+F m 2) /(R 1+R 2)
\end{gathered}
$$

$\mathrm{Fm}=$ Flux generated in inner magnet SORBLDC

$\mathrm{Fm} 1=$ Flux generated in inner magnet of DORBLDC

Fm2 = Flux generated in outer magnet of DORBLDC

$\mathrm{R} \quad=$ Rotor Reluctance of SORBLDC

$\mathrm{R}_{1} \quad=$ Inner Rotor Reluctance of DORBLDC

$\mathrm{R}_{2}=$ Outer Rotor Reluctance of DORBLDC

The magnetic equivalent circuit shows components inside conventional and proposed model by changing them into voltage source, current source, and resistor. Figure 6 shows the equivalent magnetic circuit of conventional model and proposed a model. Based on figure 6 , we can see that proposed model has higher generated flux due to mutual magnetic coupling created between outer rotor with the inner rotor to improve magnetic flux intensity by making both of them as an individual part of the machine. Therefore, the magnetic flux generated by both of individual part is amplified.

\subsection{Finite Element Analysis (FEA) Method}

Designing of the proposed model in JMAG Geometry Editor is the first step applied in the simulation of FEA analysis. Each part of the model is designed based on its parameters set for the machine. Model of the proposed machine updated in the JMAG software and configuration material for each part is done such as the type of material and its properties. Several conditions applied to each component such as rotational motion, torque nodal force, and FEM coil. Then, FEM coil circuit is constructed by setting the parameters such as resistance and voltage or current source. The setting of the windings and resistance of each coil followed with size and type of mesh to the model. Finally, simulation of the model is initialized as the steps are completed. Figure 7 shows the steps taken in FEA simulation. 


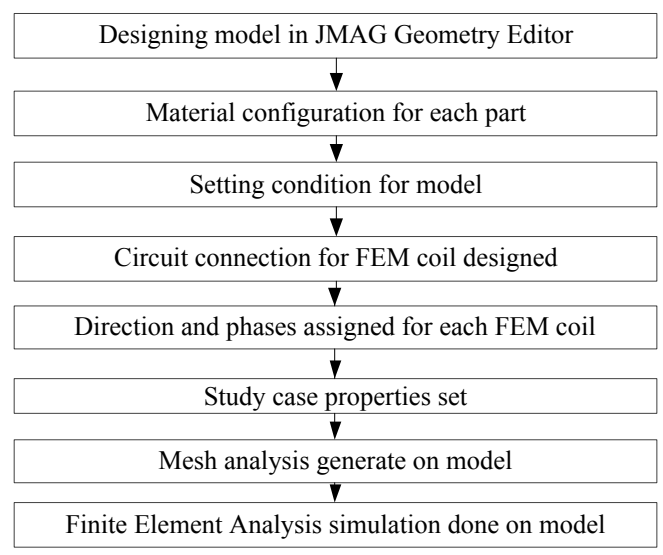

Fig. 7. Finite Element Analysis Flowchart

\section{Result and Discussion}

\subsection{Magnetic Flux Analysis between SORBLDC and DORBLDC}

Based on figure 8, the magnetic flux of amplitude for proposed model is higher than a conventional model. This shows that the proposed model has the highest magnetic flux density in the machine structure even the volume is kept the same with the conventional model. Furthermore, both of the models generates its magnetic flux pattern closing to the sinusoidal waveform which shows similarity to three-phase power supply wave. Other than that, generating magnetic flux waveform closer to sinusoidal pattern shows that harmonic occur in the machine is minimum. Torque performance analysis is done check whether the new proposed model improved the machine performance in terms of peak amplitude value. Having high generated torque of a machine enables the bike to operate under harsh road conditions such as high slope road that requires a big amount of energy to support the weight of the user and bike to accelerate uphill. In this research the torque evaluation is conducted to both models. Figure 9 shows the generated torque by both models. As shown in the graph, the proposed model generate the highest peak torque and conventional show the lower. This shows that by increasing the magnetic flux in the machine, the torque performance of the motor which increases the performance of the bike. E-bike application requires a motor that able to operate constant torque and power which show the same operation as an ICE engine with gears. The speed-torque graph of ICE engine has high starting torque and decrease as speed increases. Therefore, speed torque evaluation is done to both models in order to check whether the machine can operate same as ICE engine. Figure 10 shows the speed torque graph of the conventional and proposed model. The speed-torque graph of both models shows similarities to ICE engine speed torque. Therefore, the machine can operate for e-bike application. Based on Figure 10, the torque produced by proposed model is stable torque-speed characteristics than a conventional model. Even though SORBLDC produces high starting torque than DORBLDC, the proposed model generate higher and more stable torque-speed characteristics as the motor operate at higher speed. Therefore, the high torque produced for tougher road conditions such as uphill can be done by the proposed motor and improve the performance of the bike. 


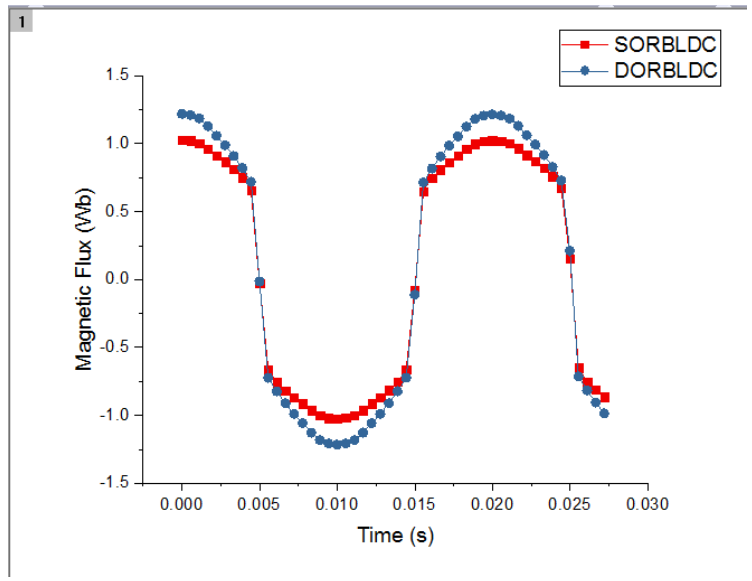

Fig. 8. Magnetic flux output of both model

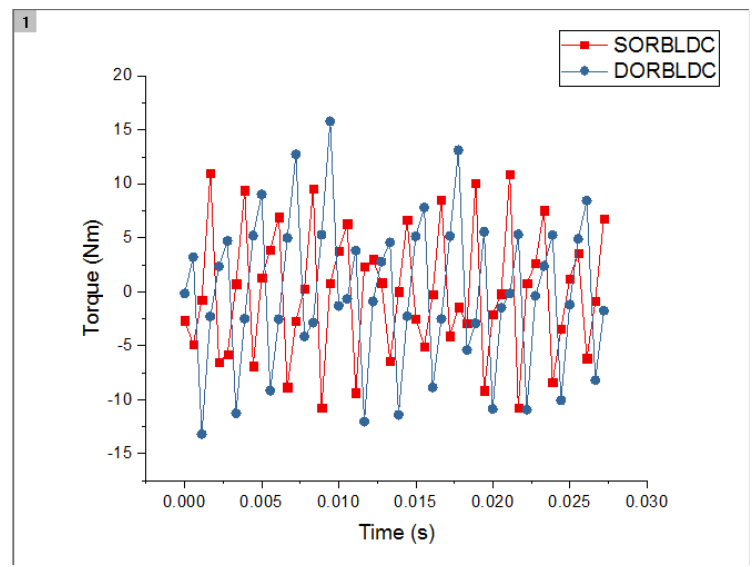

Fig. 9. Torque output of SORBLDC and DORBLDC

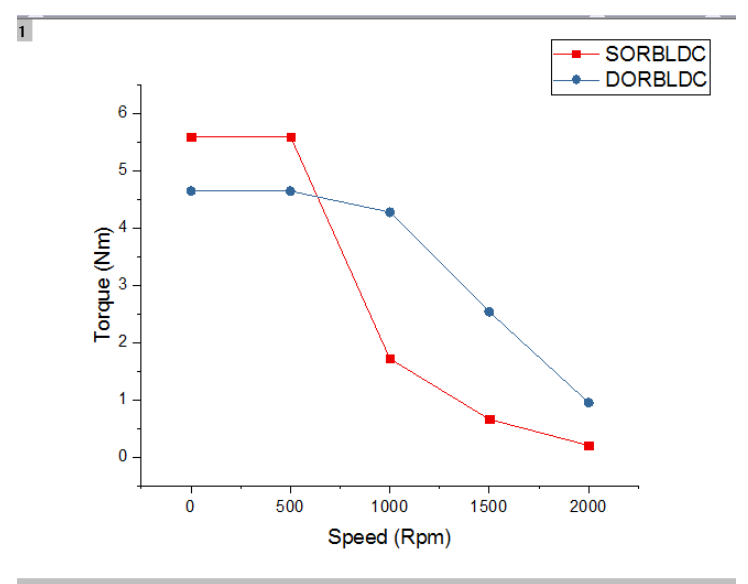

Fig. 10. Speed Torque Analysis of SORBLDC and DORBLDC 
In order to evaluate constant torque and power region of a machine, speed power analysis is conducted. The maximum speed of a machine can be identified through constant power region. Figure 11 shows speed power efficiency of SORBLDC and DORBLDC. Based on Figure 11, the speed power graph of both models shows similarities to ICE engine speed power. Therefore, the machine can operate for e-bike application. SORBLDC model shows higher power efficiency during starting speed of the machine that DORBLDC model. However, DORBLDC model shows longest constant power region which means that the machine able to operate effectively at higher speed range before losing its power at the machine speed limit which is $2000 \mathrm{rpm}$.

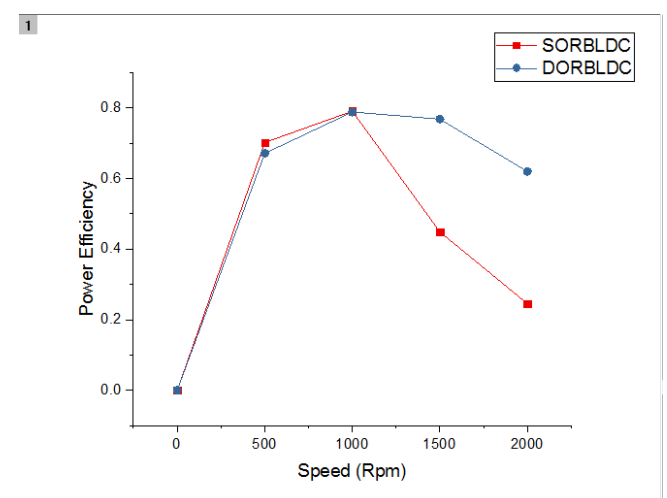

Fig. 11. Speed Power Efficiency of SORBLDC and DORBLDC

\subsection{Comparative Motor Constant Square Density Evaluation}

In this section, both models are being compared by evaluating each $G$ value. The volume of both models are calculated by multiplying the cross-sectional area of the machine with the stack length set. In the Motor Constant Square, Density equation contains parameters of a machine such as the power of the motor, torque constant, motor constant and volume of the machine. All of the parameters are calculated and tabulated in Table 2. All of the variables that needed to use to calculate the $G$ value is formed in Table 3 . From the calculated and analyzed result in Table 3, it shows that double outer rotor BLDC motor (DORBLDC) produces higher motor constant square density than single rotor BLDC motor (SORBLDC). To avoid any error in evaluating $G$ value of both model, the volume and outer radius of the machine are kept the same value. This is to ensure that the performance of both machines can be compared to the same size. From the table 3, we can see that motor constant square density of the proposed model is higher than a conventional model. The proposed model has higher $G$ value than conventional because of having more magnet reluctance in parallel in the magnetic circuit that causes improvement flux density across the machine. It shows that the proposed model design improves the torque density of the machine whereby the performance of the motor increase as the speed of the motor increased. The difference $\mathrm{G}$ between DORBLDC and SORBLDC at $2000 \mathrm{rpm}$ is: 
Table 2. Parameters of both model

\begin{tabular}{|l|l|l|}
\hline Parameter & Conventional Model & Proposed model \\
\hline $\begin{array}{l}\text { Stack length } \\
(\mathrm{mm})\end{array}$ & 50 & 50 \\
\hline Volume $\left(\mathrm{mm}^{3}\right)$ & $356,415.35$ & $311,794.70$ \\
\hline Max. Current (A) & 27 & 27 \\
\hline
\end{tabular}

Table 3. G value of both model

\begin{tabular}{|l|l|l|l|l|l|l|l|l|}
\hline \multirow{2}{*}{$\begin{array}{l}\text { Speed } \\
(\mathrm{rpm})\end{array}$} & \multicolumn{6}{|l|}{ Conventional Model } & \multicolumn{2}{l|}{ Proposed Model } \\
\cline { 2 - 9 } & $\mathrm{T}_{\text {avg }}$ & $\mathrm{K}_{\mathrm{t}}$ & $\mathrm{K}_{\mathrm{m}}$ & $\mathrm{G}$ & $\mathrm{T}_{\text {avg }}$ & $\mathrm{K}_{\mathrm{t}}$ & $\mathrm{K}_{\mathrm{m}}$ & $\mathrm{G}$ \\
\hline 0 & 5.595 & 0.155 & 4.90842 & 1.37716 & 4.6440 & 0.1290 & 3.38089 & $1.08433 \mathrm{E}-$ \\
& 685 & 436 & $\mathrm{E}-05$ & $\mathrm{E}-10$ & 6 & 02 & $\mathrm{E}-05$ & 10 \\
\hline 500 & 5.595 & 0.155 & 4.90842 & 1.37716 & 4.6440 & 0.1290 & 3.38089 & $1.08433 \mathrm{E}-$ \\
& 685 & 436 & $\mathrm{E}-05$ & $\mathrm{E}-10$ & 6 & 02 & $\mathrm{E}-05$ & 10 \\
\hline 1000 & 1.718 & 0.047 & 4.62976 & 1.29898 & 4.2762 & 0.1187 & 2.86654 & $9.19367 \mathrm{E}-$ \\
& 549 & 737 & $\mathrm{E}-06$ & $\mathrm{E}-11$ & 33 & 84 & $\mathrm{E}-05$ & 11 \\
\hline 1500 & 0.661 & 0.018 & 6.86016 & 1.92477 & 2.5382 & 0.0705 & 1.00994 & $3.23913 \mathrm{E}-$ \\
& 53 & 376 & $\mathrm{E}-07$ & $\mathrm{E}-12$ & 3 & 06 & $\mathrm{E}-05$ & 11 \\
\hline 2000 & 0.205 & 0.005 & 6.65162 & 1.86625 & 0.9520 & 0.0264 & 1.42093 & $4.55726 \mathrm{E}-$ \\
& 99 & 722 & $\mathrm{E}-08$ & $\mathrm{E}-13$ & 7 & 46 & $\mathrm{E}-06$ & 12 \\
\hline
\end{tabular}

G value improvement between SORBLDC and DORBLDC $=4.55726 \mathrm{E}-12 / 1.86625 \mathrm{E}-13$

$$
=24.41929702 \%
$$

\section{Conclusions}

In the conventional model, the generated magnetic flux across the machine can't improve as the structure of the model are kept the same. Although, V shape PM improved reluctance torque of the machine, the required space needed to mount the PM is high. Therefore, new proposed design by applying extra outer rotor layer to the conventional model able to reduce space for the magnet and produced high magnetic flux density due to amplifying generate flux produced by the inner rotor. In this research project, the proposed DORBLDC with mounted PM operate more effectively than conventional model such as improvement the motor constant square density by $24.42 \%$. Although the proposed design has few weaknesses in terms of peak torque in speed torque graph, starting power amplitude in speed power graph. However, the benefit of the proposed model such as higher magnetic flux density and longest constant torque speed characteristics than the conventional model. This shows that the proposed model is suitable for E-bike application.

\section{References}

1. J. Zhao, Y. Yangwei, Brushless DC Motor Fundamentals Application Note, MPS, Futur. 
Analog IC Technol., pp. 7-8, (2011)

2. C. Aravind, L. Pin Wen, Design Evaluations of the New Double Rotor Interior Permanent Magnet Machine, Journal of Engineering Science and Technology, 2, no. 2, pp. 73-84, (2015)

3. C. C. Hwang, S. M. Chang, C. T. Pan, T. Y. Chang, Estimation of parameters of interior permanent magnet synchronous motors, J. Magn. Magn. Mater., 239, no. 1-3, pp. 600-603, 2002

4. R. Zhang, X. Zhu, L. Chen, Design of a new magnetic-planetary-geared outer-rotor permanent-magnet brushless motor for electric vehicles, $201417^{\text {th }}$ Int. Conf. Electr. Mach. Syst. ICEMS 2014, pp. 658-663, (2015)

5. C. M. Chang, K. S. Yang, System integration and power flow management for the enginegenerator operation of a range-extended electric vehicle, 2013 World Electric. Veh. Symp. Exhib. EVS 2014, pp. 1-10, (2014)

6. M. Cheng, C. C. Chan, General Requirement of Traction Motor Drives, Encycl. Automotive. Eng., pp. 1-18, (2014).

7. M.Norrhisam, Aravind CV, S.Khodijah, N.F.Mailah, Unified control structure of multi-type interior permanent magnet motor, Journal of Engineering Sciecnes and Technology, 10, no.3 pp.322-339

8. B. Marthandam, K. Latha, Analysis of V-Shaped Interior Permanent Magnet Synchronous Motor for Low Torque Ripple, 3, no. 3, pp. 498-501, (2014)

9. C. Aravind, A. Saja, Vijayashankar, Design Evaluation of Double Rotor Switched Reluctance Machine, Journal of Engineering Science and Technology, 3, no. 1, pp. 16-26, (2016)

10. C. V. Aravind, I. Grace, T. Rozita, R. Rajparthiban, R. Rajprasad Y. V. Wong, Universal computer aided design for electrical machines, IEEE $8^{\text {th }}$ International Colloquium on Signal Processing and its Applications, Melaka, 2012, pp. 99-104, (2012) doi: 10.1109/CSPA.2012.6194699 